\title{
Aquaporin 1a expression in gill, intestine, and kidney of the euryhaline silver sea bream
}

\section{Eddie E. Deane, James C. Y. Luk and Norman Y. S. Woo*}

School of Life Sciences, The Chinese University of Hong Kong, Hong Kong, China

\section{Edited by:}

Steffen Madsen, University of

Southern Denmark, Denmark

\section{Reviewed by:}

Steffen Madsen, University of Southern Denmark, Denmark Joan Cerda, Institut de Recerca i Tecnologia Agroalimentaries, Spain

${ }^{*}$ Correspondence:

Norman Y. S. Woo, School of Life Sciences, The Chinese University of Hong Kong, Shatin, New Territories, Hong Kong, China.

e-mail:normanwoo@cuhk.edu.hk
This study aimed to investigate the effects of chronic salinity acclimation, abrupt salinity transfer, and cortisol administration on aquaporin 1 (AQP1) expression in gill, intestine, and kidney of silver sea bream (Sparus sarba). An AQP1a cDNA was cloned and found to share $83-96 \%$ amino acid sequence identity with AQP1 genes from several fish species. Tissue distribution studies of AQP1a mRNA demonstrated that it was expressed in gill, liver, intestine, rectum, kidney, heart, urinary bladder, and whole blood. Semi-quantitative RT-PCR analysis was used to measure AQP1a transcript abundance in sea bream that were acclimated to salinity conditions of $0,6,12,33,50$, and $70 \mathrm{ppt}$ for 1 month. The abundance of gill AQP1a transcript was highest in sea bream acclimated to 0 ppt whereas no differences were found among 0-50 ppt groups. For intestine, the highest AQP1a transcript amounts were found in sea bream acclimated to 12 and $70 \mathrm{ppt}$ whereas the transcript abundance of kidney AQP1a was found to be unchanged amongst the different salinity groups. To investigate the effects of acute salinity alterations on AQP1a expression, sea bream were abruptly transferred from 33 to 6 ppt. For intestine AQP1a levels were altered at different times, post transfer, but remained unchanged in gill and kidney. To study the effects of cortisol on AQP1a expression, sea bream were administered a single dose of cortisol followed by a 3-day acclimation to either 33 or $6 \mathrm{ppt}$. The findings from this experiment demonstrated that cortisol administration resulted in alterations of AQP1a transcript in gill and intestine but not in kidney.

Keywords: fish, aquaporin, gene, gill, intestine, kidney, salinity, cortisol

\section{INTRODUCTION}

The aquaporin family consists of relatively small intrinsic membrane proteins that are hydrophobic and are important in a range of physiological processes in living organisms, including water and solute transport (Borgnia et al., 1999). The diversity of aquaporins has been well reviewed and phylogenetic analysis suggests that these proteins can be grouped into three subfamilies (Heymann and Engel, 1999; Verkman and Mitra, 2000; Zardoya, 2005; Campbell et al., 2008; Ishibashi et al., 2009). The subfamily "aquaporins" are highly selective for the passage of water and are comprised of AQPs $0,1,2,4,5,6$, and 8 whereas the subfamily "aquaglyceroporins" allows for the passage of water, glycerol, and solutes such as urea and are comprised of AQPs 3, 7, 9, and 10. A third, less studied subfamily, designated as "superaquaporins" are comprised of AQPs 11 and 12 that are subcellular and have low amino acid homology to other aquaporins. Whilst most studies on aquaporins have been performed, using mammalian models, teleosts also represent excellent animal models in which to investigate their regulation and expression due to the continuous requirement toward maintaining both ion and water homeostasis. Several aquaporin families have been identified and studied, in fish, including AQP0 (killifish, Virkki et al., 2001), AQP8 (European eel, Cutler et al., 2009; Atlantic salmon, Tipsmark et al., 2010), AQP10 (Atlantic salmon, Tipsmark et al., 2010), and AQP3 from European eel (Cutler and Cramb, 2002; Lignot et al., 2002), Mozambique tilapia
(Watanabe et al., 2005), Japanese dace (Hirata et al., 2003), and silver sea bream (Deane and Woo, 2006a).

Whilst several aquaporin families are known to exist, in fish, the most ubiquitous and extensively studied family is AQP1 and topological studies have revealed that this is a tetrameric protein consisting of six transmembrane spanning domains that are joined by two hydrophobic loops to form the classic hour-glass shaped structure (Jung et al., 1994). For teleosts the AQP1 family has been studied in Japanese eel (Aoki et al., 2003), European eel (Martinez et al., 2005a,b,c), black porgy (An et al., 2008), gilthead sea bream (Fabra et al., 2005, 2006; Raldúa et al., 2008) zebrafish and Senegalese sole (Tingaud-Sequeira et al., 2008), and Atlantic salmon (Tipsmark et al., 2010). Aquaporin regulation and expression studies in gill, intestine, and kidney have also provided for important insights on water transport in fish (Cutler and Cramb, 2000). In terms of understanding the effects of altered salinity conditions on water regulation, AQP1 expression profiles from several fish have been reported. For Atlantic salmon the expression profiles of AQP1a and AQP1b, were assessed during freshwater and seawater acclimation (Tipsmark et al., 2010). The findings from this study demonstrated that $\mathrm{AQP} 1$ a transcript amounts were highest in gill following freshwater acclimation whereas amounts of this transcript were highest in kidney and middle intestine following seawater acclimation. The transcript profiles of Atlantic salmon $\mathrm{AQP} 1 \mathrm{a}$ and AQP1b were also assessed after rapid transfer from 
freshwater to seawater and it was found that AQP1a amounts were increased in the middle intestine and kidney whereas AQP1b transcript amounts increased in middle intestine and gill but decreased in kidney within the first 8 days following transfer (Tipsmark et al., 2010). The effects of chronic seawater and freshwater salinity acclimation on gill and intestinal AQP1 expression was reported for black porgy (An et al., 2008) and for both tissues the highest amounts of AQP1 transcript was found in fish acclimated to freshwater conditions. For European eel, intestinal AQP1 mRNA expression, of seawater acclimated yellow and silver eels, was found to be higher than eels that were acclimated to freshwater (Martinez et al., 2005b). Similar observations were also found for intestinal AQP1 in Japanese eels (Aoki et al., 2003), sea bass (Giffard-Mena et al., 2007), and gilthead sea bream (Raldúa et al., 2008). By using Northern blot analysis, transcript abundance of AQP1, AQP1dup, and AQPe were shown to be significantly reduced in renal tissue of yellow eels after transfer from seawater to freshwater. Conversely, renal expression levels of these aquaporins in silver eels were determined to be independent of salinity alterations (Martinez et al., 2005a).

The endocrine system is critical for regulating teleostean osmoregulatory function and amongst the plethora of hormones that have been investigated cortisol has received much attention due to its key role as a "seawater adapting hormone" (McCormick, 1995; Mommsen et al., 1999). Cortisol is a corticosteroid hormone which is synthesized by the interrenal tissue of teleost fish and is known to regulate hypoosmoregulatory processes via various ionexchange mechanisms (McCormick, 1995). To date most of our knowledge concerning the role and importance of corticosteroids, on aquaporin expression, has been derived from studies on mammals (Tanaka et al., 1997; Wintour et al., 1998; Liu et al., 2003; Stoenoiu et al., 2003). Presently there is a paucity of information regarding the role of cortisol on fish aquaporin regulation and thus far our knowledge has been confined to a study on the freshwater yellow European eel where it was found that the administration of cortisol increased intestinal AQP1 expression levels (Martinez et al., 2005a). Interestingly AQPe, was found to be unresponsive to cortisol treatment in kidney and intestine of European eels (Martinez et al., 2005a,b) whereas another aquaglyceroporin (AQP3) was found to be significantly downregulated in branchial tissues, following cortisol infusion (Cutler et al., 2007). It is clear that studies concerning the role and importance of cortisol, on teleost aquaporin expression, are certainly warranted.

As part of our investigations into understanding osmoregulatory strategies for euryhaline fish we report on the cloning of a silver sea bream (Sparus sarba) AQP1a and its expression profile during chronic salinity acclimation and after an abrupt salinity transfer. As our knowledge regarding the influence of hormones on aquaporin expression in fish is still in its infancy we have also undertaken experiments aimed at defining the importance of cortisol on silver sea bream AQP1a expression.

\section{MATERIALS AND METHODS \\ FISH AND SALINITY ACCLIMATION}

Silver sea bream (S. sarba) weighing 100-150 g, were purchased from a local fish farm, and were kept in a recirculating seawater
(33 ppt) system in the Simon F. S. Li Marine Science Laboratory, Chinese University of Hong Kong for not less than 3 weeks to allow recovery from transportation stress. The fish were then divided into six groups randomly $(n=7)$ and put into six 1 ton seawater (33 ppt) tanks with individual recirculating filter systems. The salinity of each tank was adjusted to freshwater ( $0 \mathrm{ppt})$, hypoosmotic (6 ppt), isoosmotic (12 ppt), seawater (33 ppt), hypersaline (50 ppt), or extreme hypersaline (70 ppt) by flushing gradually with either tap water or hypersaline water. These salinity adjustments were completed within 7 days and the fish were acclimated for a further 28 days at their respective final salinities. The fish were fed ad libitum with fish meal pellets (Woo and Kelly, 1995) throughout the experiment and feeding was terminated $24 \mathrm{~h}$ before fish were sacrificed. Silver sea bream were removed from the tanks and were firstly anesthetized by MS-222 (Sigma, USA). Perfusion was used to remove the blood from gills and kidney by inserting a PE-50 polyethylene cannula (Clay-Adams, USA) filled with heparinized physiological saline [composition in mmol/L: $\mathrm{NaCl}, 1.43 ; \mathrm{KCl}, 3.35 ; \mathrm{Na}_{2} \mathrm{HPO}_{4}, 1.4 ; \mathrm{MgSO}_{4} \cdot 7 \mathrm{H}_{2} \mathrm{O}, 4 ; \mathrm{CaCl}_{2}, 1.3$; $\left(\mathrm{NH}_{4}\right)_{2} \mathrm{SO}_{4}, 0.3 ; \mathrm{KH}_{2} \mathrm{PO}_{4}, 0.3$; glucose, 5.6] into the first afferent branchial artery and then attaching the tube to a peristaltic pump. The aorta, slightly anterior to bulbus arteriosus, was tied off by a silk suture and an incision was made between the bulbus arteriosus and the heart to allow the blood/perfusate mixture to run out. The saline was kept in an ice-bath throughout the perfusion operation. Perfusion was also performed via the dorsal aorta on several occasions when blood inside the kidney had not been removed properly by the branchial afferent artery perfusion. The perfusion operation was deemed complete when the red color of the organs disappeared. For subsequent mRNA studies small pieces of gill, intestine, and kidney from all salinity acclimated groups were placed into $1 \mathrm{ml}$ of Tri-reagent (Molecular Research Center, USA). For tissue mRNA studies, samples of gill, intestine, kidney, brain, heart, liver, rectum, urinary bladder, and whole blood were also kept in $1 \mathrm{ml}$ Tri-reagent and all samples were then stored at $-70^{\circ} \mathrm{C}$.

\section{ABRUPT SALINITY TRANSFER}

Silver sea bream weighing 100-150 g, were purchased and maintained in seawater as described above. To perform an abrupt salinity transfer, twelve 1 ton tanks, with independent recirculating systems, were prepared such that six of these contained seawater (33 ppt), and six contained hypoosmotic (6 ppt) water. Groups of randomly selected fish $(n=7)$ were abruptly transferred from seawater conditions to either seawater or hypoosmotic conditions and sacrificed at 2, 6, 12, 72, and $168 \mathrm{~h}$ following transfer. Pretransferred fish were used as time $=0 \mathrm{~h}$ group and feeding was terminated $24 \mathrm{~h}$ prior to performing the experiment. To assess mRNA amounts, small pieces of gill, intestine, and kidney tissues were prepared and collected as described above.

\section{CORTISOL TREATMENT}

For these sets of experiments silver sea bream weighing between 50 and $100 \mathrm{~g}$, were purchased from a local fish farm and maintained in seawater as described above. In order to minimize stress, the administration, and dosage of cortisol followed previously described procedures (Pelis and McCormick, 2001). Silver sea 
bream were initially divided into two groups $(n=14)$ and each group of fish were lightly anesthetized with MS-222 (Sigma), weighed, and either intraperitoneally injected with $10 \mu \mathrm{l} / \mathrm{g}$ body weight of a slow releasing oil implant (mixture 1:1 coconut oil and olive oil) or an oil implant containing cortisol (hydrocortisone, Sigma) to a final amount that was equal to $50 \mu \mathrm{g} / \mathrm{g}$ body weight. The subsequent abrupt transfer followed the procedure as described for gilthead sea bream (Sangio-Alvarellos et al., 2006) whereby after 3 days, following implantation, each treatment group was further divided into two sub-groups $(n=7)$, transferred to $6 \mathrm{ppt}$ or to $33 \mathrm{ppt}$ (transfer control), and sampled at 3 days post transfer. Serum cortisol levels were measured using an ELISA kit purchased from Cayman Chemical Company (Michigan USA). The cortisol implantation procedure used was sufficient to maintain a state of hypercortisolemia as the average levels of cortisol were measured (as $\mathrm{ng} / \mathrm{ml}$ ) and found to be $11.4 \pm 5.4$ (33 ppt transfer/oil), $232 \pm 49.9$ (33 ppt transfer/cortisol), $24.11 \pm 8.7$ (6 ppt transfer/oil), and $186 \pm 31.4$ (6 ppt transfer/cortisol).

\section{CLONING AND SEQUENCE ANALYSIS OF SILVER SEA BREAM AQP1}

Total RNA from silver sea bream tissues were prepared using Tri-Reagent (Molecular Probes), treated with DNase I and then quantified spectrophotometrically. Total RNA ( $1 \mu \mathrm{g})$ was mixed with $0.5 \mu \mathrm{g}$ oligo-dT primer (Proligo) and MMLV reverse transcriptase (InVitrogen) and first strand cDNA synthesis was allowed to proceed at $42^{\circ} \mathrm{C}$ for $2 \mathrm{~h}$. Following synthesis the reaction mix was incubated at $70^{\circ} \mathrm{C}$ for $10 \mathrm{~min}$ and the cDNA preparations were stored at $-20^{\circ} \mathrm{C}$. In order to perform PCR amplification of AQP1 gene fragments we compared available fish AQP1 gene sequences on GenBank and identified two highly conserved regions. From the sequence information of these regions two sense primers designated as A1S1 (5'-CTTCTGGAGGGCCGTTCT- $\left.3^{\prime}\right)$ and A1S2 (5'-TGTTCAAGGCGGTCATGTA- $\left.3^{\prime}\right)$ and three antisense primers designated as A1AS1 (5'-ATGTACATGACCGCCTTGA$\left.3^{\prime}\right)$, A1AS2 (5'-CACCCAGTACACCCAGTGGT-3'), and A1AS3 (5'-GGGAAGTCGTCGAATTTGG-3') were synthesized (Genset, Singapore). For amplification of silver sea bream AQP1, PCR reactions were carried out in a volume of $25 \mu \mathrm{l}$ containing $2 \mu \mathrm{l}$ first strand cDNA, $5 \mathrm{U}$ of Taq polymerase (Promega), $0.25 \mu \mathrm{l} \mathrm{dNTP}$ mix $(10 \mathrm{mM}), 2.5 \mu \mathrm{l} \mathrm{MgCl}_{2}(25 \mathrm{mM}), 2.5 \mu \mathrm{l}$ of PCR reaction buffer, and $0.8 \mathrm{mM}$ of each primer. PCR amplifications were performed using an Eppendorf 9600 Thermal Cycler (Eppendorf, Germany) with a gradient of annealing temperatures from 45 to $60^{\circ} \mathrm{C}$. Subsequent PCR reactions, cloning of gene fragments, and sequencing of putative clones were performed as described previously (Deane and Woo, 2004). A single DNA fragment of about $690 \mathrm{bp}$ was obtained with the primer combination of A1S1 and A1AS3 under a high stringency annealing temperature of $60^{\circ} \mathrm{C}$. As initial sequence analysis demonstrated that this fragment had a high degree of homology to known fish AQP1 sequences we proceeded to isolate the entire open reading frame (ORF) using $5^{\prime}$ and $3^{\prime}$ RACE systems (InVitrogen) and the cloned fragments were fully sequenced. The sequence data was analyzed using Clustal $\mathrm{W}^{1}$ and Expasy $^{2}$.

${ }^{1}$ www.ebi.ac.uk

${ }^{2}$ www.expasy.org

\section{SEMI-QUANTITATIVE AQP1A RT-PCR}

Extraction of total RNA from each sea bream tissue and reverse transcription were performed as described above. Reverse transcriptase products of negative control for each tissue were also prepared with the same amount of RNA without MMLV reverse transcriptase. The primers used for RT-PCR were A1S1 and A1AS3 as described above and each RT-PCR reaction mix $(25 \mu \mathrm{l})$ contained $1 \times$ PCR buffer, $0.5 \mathrm{mM}$ each dNTP, $2.5 \mathrm{mM} \mathrm{MgCl}_{2}$, $0.8 \mathrm{mM}$ each primer, $0.5 \mathrm{U}$ of Taq polymerase. Polymerase chain reaction was performed using an Eppendorf 9600 Thermal Cycler with parameters of $94^{\circ} \mathrm{C}$ for $30 \mathrm{~s}, 60^{\circ} \mathrm{C}$ for $30 \mathrm{~s}$, and $72^{\circ} \mathrm{C}$ for $1 \mathrm{~min}$. The PCR products were separated on $2 \%(\mathrm{w} / \mathrm{v})$ agarose gel (with ethidium bromide) and the band intensities were quantified with a Gel-Doc 1000 system (Bio-Rad) and analyzed using Quantity One Molecular Analyst Software (Bio-Rad). The optimum cycle number was obtained by plotting an amplification profile, with the optimum cycle number being the mid cycle number of the exponential phase in the amplification profile for all organs. The optimal cycle number used for tissue distribution analysis was 30 cycles as it fell at the exponential phases for most tissues tested. After validation, PCR was performed with the same profile for 30 cycles and as a normalization control, primers for $\beta$ actin were used according to previously described procedures for sea bream (Deane et al., 2002).

\section{STATISTICAL ANALYSIS}

All data were expressed as mean values \pm SEM. Data from each chronic salinity acclimation and cortisol treatment groups were subjected to a one-way analysis of variance (ANOVA), followed by a Tukey's test (SPSS) with $p<0.05$ to delineate significance. Data from abrupt transfer experiments were subjected to twoway ANOVA and to delineate the significance of the interaction between salinity and time.

\section{RESULTS \\ CHARACTERIZATION OF SILVER SEA BREAM AQP1a cDNA}

The silver sea bream AQP1 cDNA clone was 904 base pairs (bp) containing $51 \mathrm{bp}$ of a $5^{\prime}$-untranslated region (UTR), $774 \mathrm{bp}$ of an ORF, and $79 \mathrm{bp}$ of $3^{\prime}$-UTR. The nucleotide sequence encodes a protein with 258 amino acids with a calculated mass of $27.1 \mathrm{kDa}$ and has been submitted on GenBank under accession number JF803845. Importantly the two channel-forming NPA (asparagine-proline-alanine) signature motifs are located at amino acid positions 67-69 and 181-183. Sequence comparison of the encoded protein, with known AQP1 proteins, also indicated the presence of amino acid residues of the pore-forming region at positions 56 (phenylalanine), 180 (histidine), and 195 (arginine). A cysteine residue is located before the second NPA motif at position 178, which is the potential site responsible for the inhibition of water permeability by mercurial compounds. The silver sea bream AQP1 protein shared high levels of amino acid identity with AQP1a from gilthead sea bream (97.7\%) black porgy (95.8\%), and European eel (83.6\%; Figure 1). Also the cloned silver sea bream AQP1 amino acid sequence shares relatively low identity to AQP1b of the gilthead sea bream (56\%) and human AQP1 (57.8\%). 


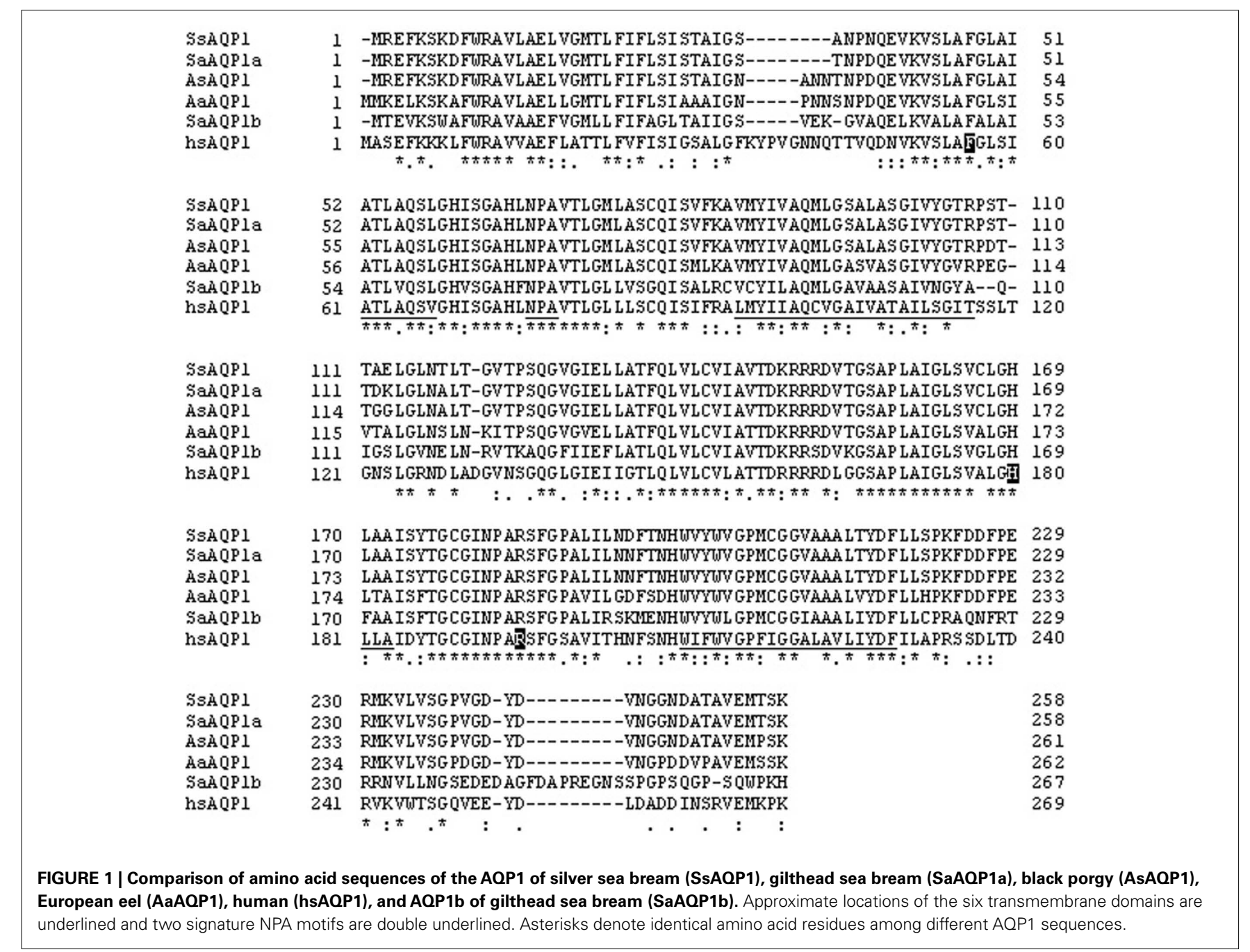

\section{TISSUE DISTRIBUTION OF SILVER SEA BREAM AQP1a TRANSCRIPT}

Using RT-PCR it was found that the AQP1a transcript was expressed in gill, liver, intestine, rectum, kidney, heart, urinary bladder, and whole blood but not in brain tissue of silver sea bream (Figure 2). For each tissue studied no amplified signals were detected in MMLV-negative controls therefore eliminating the possibility of false positive results due to genomic DNA contamination.

\section{EFFECTS OF CHRONIC SALINITY ACCLIMATION ON AOP1a TRANSCRIPT ABUNDANCE}

Silver sea bream were acclimated, for 1 month, to salinities ranging from freshwater $(0 \mathrm{ppt})$ to extreme hypersaline (70 ppt) and transcript amounts of AQP1a were semi-quantified using RT-PCR. In gill, the abundance of AQP1 a transcript was found to be the highest in freshwater acclimated sea bream, whereas no significant difference in the expression levels was observed between 6 and $70 \mathrm{ppt}$ salinity groups (Figure 3A). For intestine, the expression of AQP1a was found to be the highest in fish that were acclimated to $70 \mathrm{ppt}$ being approximately 2.1-7.9 fold higher than amounts from sea bream acclimated between 6 and 50 ppt (Figure 3B). It was also

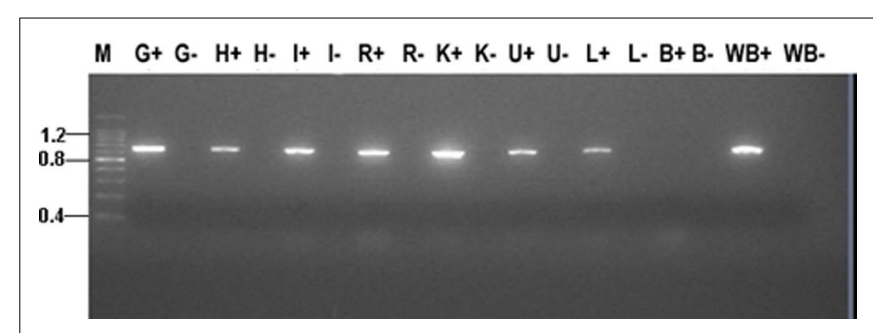

FIGURE 2 | Tissue distribution of AQP1a transcript in seawater acclimated silver sea bream. Abbreviations: $M$, molecular marker (sizes indicated kb); $G$, gill; $H$, heart; I, intestine; $R$, rectum; $K$, kidney; $U$, urinary bladder; L, liver; B, brain; and WB, whole blood. A (+) or (-) after an abbreviation indicates reverse transcriptase reactions that were performed with or without MMLV respectively.

found that intestinal AQP1a transcript abundance was increased to a smaller but significant extent in sea bream that were acclimated to an isosmotic salinity (12 ppt) when compared to levels from 0, 6, and 33 ppt acclimated sea bream (Figure 3B). In kidney, the expression level for AQP1a was not significantly different over the salinity range tested (Figure $3 \mathrm{C}$ ). 


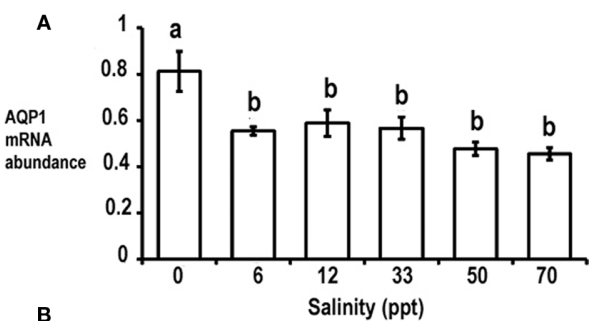

B
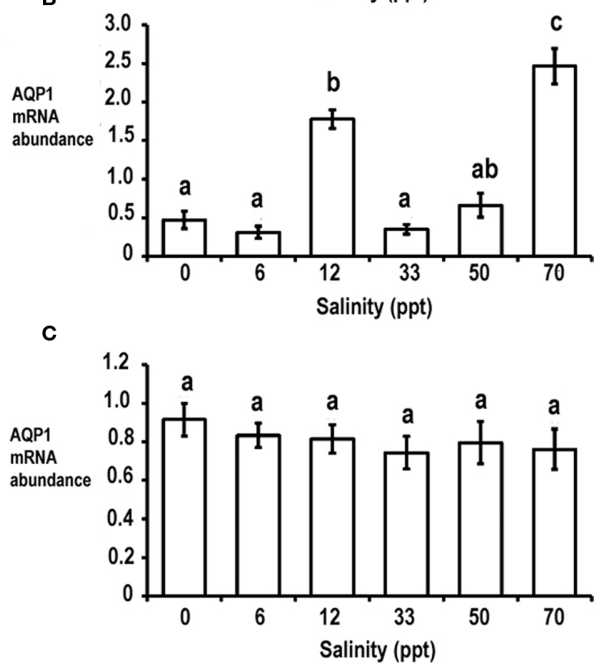

FIGURE 3 | Expression of AQP1a transcript in gill (A), intestine (B), and kidney (C) of silver sea bream that were acclimated to freshwater (0 ppt), hypoosmotic (6 ppt), isoosmotic (12 ppt), seawater (33 ppt), hypersaline (50 ppt), and extreme hypersaline (70 ppt) for 1 month. Data is presented as normalized AQP1a transcript abundance (arbitrary units) and values are expressed as mean \pm SEM $(n=7)$. Different letters above a bar denote significant differences among salinity acclimated groups $(p<0.05)$.

\section{EFFECTS OF ABRUPT SALINITY TRANSFER ON AOP1a TRANSCRIPT ABUNDANCE}

To study the effects of acute salinity change on AQP1a transcript expression, sea bream were abruptly transferred from seawater (33 ppt) to hypoosmotic (6 ppt) conditions. Gill AQP1a transcript amounts started to decline at $2 \mathrm{~h}$ post transfer and continued to fall until $12 \mathrm{~h}$ post transfer. The abundance of gill AQP1a transcript was lowest at $12 \mathrm{~h}$ post transfer as the amount of AQP1a mRNA was less than half of that found in the control transfer group at the same time point (Figure 4A). However following statistical analysis it was found that there was not a significant interaction between salinity and time for gill AQP1a transcript amounts. Intestinal AQP1a transcript abundance exhibited transient perturbations in both the hypoosmotic transferred group and the seawater transfer control group but there was not a significant interaction between time and salinity. However it was found that time following transfer had a significant effect on AQP1a transcript abundance (Figure 4B). There was no significant interaction between salinity and time for kidney AQP1a transcript amounts when compared to transfer control groups (Figure 4C).
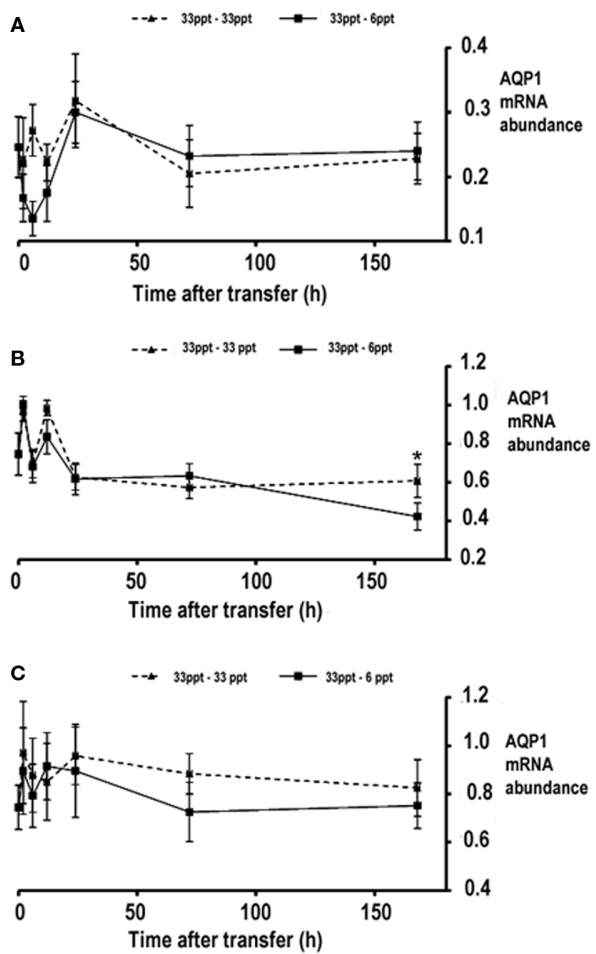

FIGURE 4 | Expression of AQP1a transcript in gill (A), intestine (B), and kidney (C) following an abrupt transfer of silver sea bream. Two

transfers were performed, a 33- to 6-ppt abrupt hypoosmotic transfer and a control 33-33 ppt transfer. Data is presented as normalized AQP1a transcript abundance (arbitrary units) and values are expressed as mean \pm SEM $(n=7-4)$. There was no significant interaction between salinity and time $(p<0.05)$.

\section{EFFECT OF CORTISOL ADMINISTRATION ON AOP1a TRANSCRIPT ABUNDANCE}

In order for us to define the importance of hormones on regulating silver sea bream AQP1a expression we performed experiments whereby silver sea bream were treated with cortisol and then transferred to either 33 or 6 ppt for 3 days. Gill AQP1a transcript abundance remained unchanged between the oil control and cortisol treated fish, regardless of salinity (Figure 5A). Administration of cortisol caused approximately a 1.8-fold increase in AQP1a transcript abundance in the intestine of sea bream acclimated to 33 and 6 ppt when compared to the oil control group (Figure 5B). Comparable to our findings with gill, cortisol treatment did not modulate the abundance of AQP1a transcript in silver sea bream kidney when compared to oil control groups (Figure 5C).

\section{DISCUSSION}

\section{AOP1a GENE CHARACTERIZATION AND TISSUE EXPRESSION}

Toward our molecular studies a cDNA of the silver sea bream AQP1 homolog was isolated and found to share high identity with several previously isolated fish AQP1 genes. Hydropathy analysis predicted that it consists of six transmembrane domains which are key characteristics found in the major intrinsic protein (MIP) 


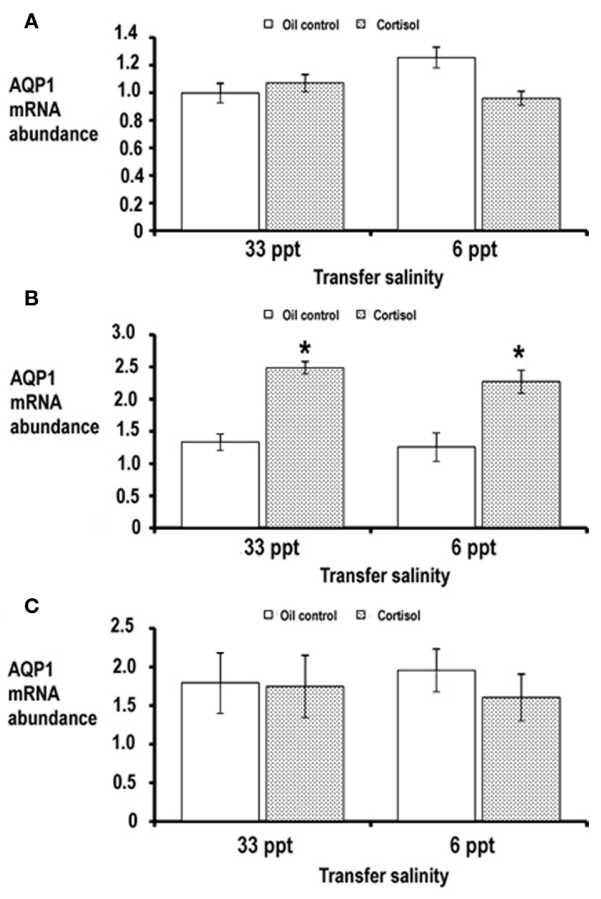

FIGURE 5 | Expression of AQP1a in gill (A), intestine (B), and kidney (C) of silver sea bream following administration of oil alone (control) or cortisol and then transferred to either $\mathbf{3 3}$ or $\mathbf{6}$ ppt salinity. Data is presented as normalized AQP1a transcript abundance (arbitrary units) and values are expressed as means $\pm \operatorname{SEM}(n=7)$. An asterisk above a bar indicates values that were found to be different between oil and cortisol treated groups $(p<0.05)$

family as well as amongst aquaporins (Borgnia et al., 1999). The repeat amino acid sequences of loops $B$ and $E$ in silver sea bream AQP1 were highly conserved with respect to other mammalian and fish AQP1 homologs and are considered to be functionally important for water permeability (Jung et al., 1994; Shi et al., 1994; Borgnia et al., 1999). Also present in loops B and E were the signature NPA motifs that appear to act mainly as size exclusion filters (De Groot and Grubmuller, 2001). The cysteine residue (amino acid 178) was also located in loop $\mathrm{E}$ at the putative mercurial inhibiting site found in human AQP1 homologs (Borgnia et al., 1999). The silver sea bream AQP1 protein shared highest levels of amino acid identity with AQP1a from gilthead sea bream $(97.7 \%)$ but relatively low identity to AQP1b of the gilthead sea bream (56\%) therefore it would seem most likely that we had isolated an AQP1a from silver sea bream. Transcript analysis revealed the presence of AQP1a in gill, heart, intestine, rectum, kidney, liver, urinary bladder, and whole blood which is in general agreement with studies in mammals (Ishibashi et al., 2009) and teleosts (Aoki et al., 2003; Martinez et al., 2005a; An et al., 2008; Raldúa et al., 2008). In contrast, AQP1 transcript was not detected in sea bream brain but has been detected in the brain of the European eel (Martinez et al., 2005a) and black porgy (An et al., 2008). Taken together these findings suggest that the tissue expression profile of AQP1 is not constant amongst different fish species.

\section{EFFECT OF CHRONIC SALINITY ACCLIMATION ON AOP1a TRANSCRIPT ABUNDANCE}

We investigated AQP 1a transcript abundance in gill, intestine, and kidney tissues taken from fish that were acclimated for 1 month to salinities ranging from freshwater $(0 \mathrm{ppt})$ to extreme hypersaline $(70 \mathrm{ppt})$. For gill, it was found that freshwater acclimation resulted in an elevated abundance of AQP1a transcript only whereas acclimation to salinities ranging from 6 to $70 \mathrm{ppt}$ did not have any significant effect on gill AQP1a expression. This finding is in general agreement with those reported for black porgy AQP1 (An et al., 2008) and Atlantic salmon AQP1b (Tipsmark et al., 2010) where transcript amounts were higher in freshwater acclimated fish compared to those maintained in seawater. The elevated AQP1a transcript abundance in freshwater acclimated silver sea bream may contribute to the higher osmotic water permeability known to occur in gills of freshwater fish (Motais and Isaia, 1972). Preliminary immunohistochemical analysis has indicated that AQP1 could be localized within the chloride cells of sea bream gill (unpublished data). Although these findings await further and more stringent confirmation it could be plausible that AQP1a may act as an exit point to release water through the basolateral membrane of these cells to the serosal fluid in order to prevent cell swelling during hypoosmotic exposure. However, we cannot overlook the possibility that AQP1a may act in concert with other AQP homologs to prevent excessive cell swelling, in gill cells, as the amounts of the AQP3 homolog have been shown to increase upon freshwater acclimation of yellow and silver European eel (Cutler and Cramb, 2002) and sea bass (Giffard-Mena et al., 2007). Also a previous study on silver sea bream found that low (6 ppt) salinity acclimation caused a four-fold increase in gill AQP3 protein amounts in comparison to fish acclimated to seawater conditions (Deane and Woo, 2006a). We also studied intestinal AQP1a expression in silver sea bream and it was found that the largest and most significant increase in intestinal AQP1a transcript occurred following acclimation to extreme hypersaline (70 ppt) conditions. With regards to the increased AQP1a transcript in $70 \mathrm{ppt}$ conditions our results generally support previous findings on European yellow and silver eels (Martinez et al., 2005b) and Japanese eels (Aoki et al., 2003) where seawater acclimation resulted in elevated AQP1 in comparison to fish acclimated to freshwater conditions. The elevated intestinal AQP1a expression would likely contribute to increased water permeability and hence a higher intestinal water absorption rate that would occur as silver sea bream were acclimated to hypersaline environments. It was also of interest that we found a significant increase in AQP1a transcript during isosmotic salinity (12 ppt) acclimation. Whilst there are no other studies that have reported similar findings we speculate that this may be a consequence of altered hormonal status in particular increased amounts of circulating growth hormone $(\mathrm{GH})$. $\mathrm{GH}$ is well established to play a key role during seawater acclimation of fish (McCormick, 1995; Sakamoto and McCormick, 2006; Deane and Woo, 2009) and in several species, mainly belonging to the Sparidae, the highest amounts of GH have been shown to occur following isosmotic salinity acclimation (Mancera et al., 1995; Deane and Woo, 2004, 2006b, 2009). As we have shown previously that isosmotic salinity acclimation 
of silver sea bream causes an increase in pituitary GH transcript then it is possible that the higher availability of $\mathrm{GH}$ could have altered intestine functions through increased water absorption and hence elevated AQP1a expression. To date no studies have examined the role and importance of GH on teleostean AQP expression and research within this context would be very informative in aiding our understanding of hormonal control of water regulation in fish. Together with the gill and intestine the kidney may also play a key role during salinity acclimation of fish particularly in relation to maintaining water balance via adjustments of glomerular filtration rate (McDonald and Grosell, 2006). Although aquaporins could be important in such processes, in the present study AQP1a transcript abundance remained unchanged in kidney from silver sea bream acclimated between freshwater to extreme hypersaline conditions. In this regard our data contrast to those reported for AQP1 from black porgy (An et al., 2008) and AQP1a from Atlantic salmon (Tipsmark et al., 2010) where transcript amounts were highest in freshwater acclimated fish in comparison to those maintained in seawater. Also renal AQP1b transcript amounts were found to be significantly lower in freshwater acclimated Atlantic salmon when compared to those maintained in seawater and indicates differences in AQP isoform expression (Tipsmark et al., 2010). The alterations in renal AQP1 expression levels, during salinity acclimation, may also exhibit species specificity as yellow and silver eels were found to have lowered or unchanged renal AQP1 transcript abundance, respectively, when fish were transferred from freshwater and acclimated to seawater for 3 weeks (Martinez et al., 2005a). Whilst we could not detect alterations in renal AQP1 for silver sea bream it is possible that other renal AQP homologs may be modulated and therefore play key roles in maintaining water balance. Indeed studies on European eel have demonstrated the existence of a renal AQP1 isoform (AQP1a) and an aquaglyceroporin (AQPe) both of which exhibit markedly higher transcription levels than AQP1 (Martinez et al., 2005a).

\section{EFFECT OF ABRUPT SALINITY TRANSFER ON AQP1a TRANSCRIPT ABUNDANCE}

To complement our chronic salinity acclimation studies we also investigated whether an abrupt transfer of silver sea bream from seawater $(33 \mathrm{ppt})$ to hypoosmotic $(6 \mathrm{ppt})$ conditions could have an effect on the transient expression of AQP1a. In gill, it was found that during the first $6 \mathrm{~h}$, following hypoosmotic transfer, the abundance of branchial AQP1a transcript was lower than the seawater transfer control group although no statistical significant differences were found between salinity and/or time. However it is apparent when comparing our AQP1a expression data from chronic salinity acclimation and abrupt transfer experiments that during the hours following abrupt transfer different data are derived. It is possible that the rapid post exposure decrease in AQP1a transcript, following abrupt hypoosmotic transfer, indicates that there was a "shut-down" of water permeation across the gill epithelium to prevent further osmotic water load entry. Indeed a previous study on isolated Japanese eel gill preparations demonstrated that water permeability decreased within the first few hours after transfer from seawater to freshwater (Ogasawara and Hirano, 1984). It was also found from studies on Japanese eel that chloride cell number and apical pit diameter were also decreased within the first $6 \mathrm{~h}$ following transfer from seawater to freshwater (Ogasawara and Hirano, 1984). Similarly, in a previous study on abrupt transferred silver sea bream a transient reduction in gill chloride cell numbers was observed after a 6-h hypoosmotic exposure (Kelly and Woo, 1999). Also the morphology of the apical membranes of gill chloride cells changed from invaginated to co-existing on the same plane or protruding above pavement cells (Kelly and Woo, 1999). Given that our preliminary immunohistochemical studies (unpublished data) indicate that the majority of AQP1 is associated within the gill chloride cell then it is plausible that the decreased AQP1a transcript, found during early hypoosmotic transfer, is most likely associated with decreased chloride cell number as well as modifications of chloride cell morphology. For intestine, AQP1a transcript amounts were observed to fluctuate quite widely during the first $12 \mathrm{~h}$ in both seawater and hypoosmotic transferred groups but stabilized at $168 \mathrm{~h}$ post transfer. After statistical analysis it was found that the abundance of intestinal AQP1a was not significantly changed due to the interaction of salinity. However it was found that time did have a significant effect on transcript amounts which suggests that AQP1a expression is prone to wide temporal fluctuations during abrupt salinity transfer. In accordance with our findings for chronic salinity acclimated silver sea bream, renal AQP1a transcript amounts were unchanged following an abrupt hypoosmotic salinity transfer. Whilst it is possible that functional protein amounts could have been altered it would appear from transcript studies that there is probably little importance for renal AQP1a during conditions that can cause rapid hydration of silver sea bream. In freshwater teleosts the glomerular filtration rate is known to be very high in order to remove excess water (Nishimura and Imai, 1982) and therefore we cannot exclude the possibility that other aquaporin homologs are involved in this process but further and more comprehensive studies will be needed to investigate this conjecture.

\section{MODULATION OF AQP1a TRANSCRIPT ABUNDANCE FOLLOWING CORTISOL ADMINISTRATION}

The final part of this study aimed to investigate whether cortisol treatment could modulate AQP1a transcript amounts in silver sea bream. The circulating levels following cortisol administration demonstrated that the fish were hypercortisolemic with serum cortisol levels approximately 8-20 fold higher than controls. Similar conditions of hypercortisolemia have been previously shown to alter expression profiles of $\mathrm{Na}^{+}-\mathrm{K}^{+}-$ATPase and stress proteins in silver sea bream (Deane et al., 1999a,b, 2006; Deane and Woo, 2011). Even though fish were in a hypercortisolemic state we did not detect any significant alterations for both branchial and renal AQP1a transcript. To date no studies have examined the importance of cortisol on branchial AQP1a expression but our data on renal AQP1a are different from those reported for European eels where cortisol treatment caused an increase in AQP1 transcript abundance (Martinez et al., 2005a). Presently we cannot explain this disparity but the functional 
role of cortisol may be dependent upon its receptors which in turn may exhibit species specificity. We did however observe that cortisol treatment caused an increase in AQP1a transcript in the intestine of silver sea bream that were transferred to either seawater or hypoosmotic conditions. A similar finding has been reported for freshwater acclimated yellow eels where cortisol treatment caused a three-fold increase in AQP1 transcript in intestinal epithelial scrapes (Martinez et al., 2005b). Cortisol has been shown to influence water transport in fish as the fluid uptake rate across the posterior intestine of Atlantic salmon was approximately doubled following cortisol treatment (Cornell et al., 1994). Also earlier studies have reported that the injection of cortisol mimicked changes in intestinal water movement during seawater transfer of European eel (Gaitskell and Chester Jones, 1970) and Japanese eel (Hirano and Utida, 1968). Therefore the findings from our study suggest that cortisol can modulate intestinal AQP1a expression in silver sea bream and this may be related to the cortisol enhanced intestinal water transport previously found in Atlantic salmon, European eel, and Japanese eel.

\section{REFERENCES}

An, K. W., Kim, N. N., and Choi, C. Y. (2008). Cloning and expression of aquaporin 1 and arginine vasotocin receptor mRNA from the black porgy, Acanthopagrus schlegeli: effect of freshwater acclimation. Fish Physiol. Biochem. 34, 185-194.

Aoki, M., Kaneko, T., Katoh, F., Hasegawa, S., Tsutsui, N., and Aida, K. (2003). Intestinal water absorption through aquaporin 1 expressed in the apical membrane of mucosal epithelial cells in seawater-adapted Japanese eel. J. Exp. Biol. 206, 3495-3595.

Borgnia, M., Nielsen, S., Engel, A., and Agre, P. (1999). Cellular and molecular biology of the aquaporin. Ann. Rev. Biochem. 68, 425-458.

Campbell, E. M., Ball, A., Hoppler, S., and Bowman, A. S. (2008). Invertebrate aquaporin: a review. J. Comp. Physiol. B 178, 935-955.

Cornell, S. C., Portesi, D. M., Veillette, P. A., Sundell, K., and Specker, J. L. (1994). Cortisol stimulates intestine fluid uptake in Atlantic salmon (Salmo salar) in the postsmolt stage. Fish Physiol. Biochem. 13, 183-190.

Cutler, C. P., and Cramb, G. (2000). "Water transport and aquaporin expression in fish," in Molecular Biology and Physiology of Solute Transport, eds S. Hohmann and S. R. Nielsen (New York: Kluwer Academic/Plenum Publishers), 433-440.

Cutler, C. P., and Cramb, G. (2002). Branchial expression of an Aquaporin 3 (AQP-3) homologue is down-regulated in the European eel (Anguilla anguilla) following seawater acclimation. J. Exp. Biol. 205, 2643-2651.

Cutler, C. P., Philips, C., and Hazon, N. (2009). Aquaporin 8 (AQP8) intestinal mRNA expression increases in response to salinity acclimation in yellow and silver European eels (Anguilla anguilla). Comp. Biochem. Physiol. 153A, S78.

Cutler, C. P., Philips, C., Hazon, N., and Cramb, G. (2007). Cortisol regulates eel (Anguilla anguilla) aquaporin 3 (AQP3) mRNA expression levels in gill. Gen. Comp. Endocrinol. 152, 310-313.

De Groot, B. L., and Grubmuller, H. (2001). Water permeation across and dynamics of aquaporin-1 and GlpF. Science 294, 2353-2357.

Deane, E. E., Kelly, S. P., Luk, J. C. Y., and Woo, N. Y. S. (2002). Chronic salinity adaptation modulates hepatic heat shock protein and insulinlike growth factor I expression in black sea bream. Mar. Biotechnol. 4 , 193-205.

Deane, E. E., Kelly, S. P., and Woo, N. Y. S. (1999a). Hormonal modulation of branchial $\mathrm{Na}^{+-} \mathrm{K}^{+-}$ATPase subunit mRNA in a marine teleost Sparus sarba. Life Sci. 64, 1819-1829.

Deane, E. E., Kelly, S. P., Lo, C. K., and Woo, N. Y. S. (1999b). Effect of growth hormone, prolactin and cortisol on hepatic HSP70 expression in a marine teleost Sparus sarba. J. Endocrinol. 161, 413-421. biological membranes: mechanism

\section{CONCLUSION}

In this study, we have shown that the expression profile of sea bream AQP1a is significantly altered during chronic salinity acclimation although abrupt salinity transfer has little effect on transcript abundance. We cannot overlook the possibility that AQP1a may only play a partial role in regulating water exchange and the importance of other AQPs need to be fully understood and investigated. Studies on the effects of hormones on AQP expression, in fish, are few and in this study we were able to demonstrate that cortisol administration can modulate AQP1a in silver sea bream intestine. However studies aimed at addressing the role of other key osmoregulatory important hormones such as prolactin, GH, and insulin-like growth factor 1 would certainly prove to be worthwhile in aiding our understanding of how fish AQPs are regulated.

\section{ACKNOWLEDGMENTS}

This research was supported by a Hong Kong Research Grants Council General Research Fund (CUHK4783/09M) awarded to Dr. N. Y. S. Woo.

Deane, E. E., and Woo, N. Y. S. (2004). Differential gene expression associated with euryhalinity in sea bream (Sparus sarba). Am. J. Physiol. 287, R1054-R1063.

Deane, E. E., and Woo, N. Y. S. (2006a). Tissue distribution, effects of salinity acclimation, and ontogeny of aquaporin 3 in the marine teleost, silver sea bream (Sparus sarba). Mar. Biotechnol. 8, 663-671.

Deane, E. E., and Woo, N. Y. S. (2006b). Molecular cloning of growth hormone from silver sea bream: effect of abiotic and biotic stress on transcriptional and translational expression. Biochem. Biophys. Res. Commun. 342, 1077-1082.

Deane, E. E., Zhou, L., and Woo N. Y. S. (2006). Cortisol can be pro- or anti-apoptotic in sea bream cells: potential role of HSP70 induction for cytoprotection. Mol. Cell. Endocrinol. 259, 57-64.

Deane, E. E., and Woo, N. Y. S. (2009). Modulation of fish growth hormone levels by salinity, temperature, pollutants and aquaculture related stress: a review. Rev. Fish Biol. Fish. 19, 97-120.

Deane, E. E., and Woo, N. Y. S. (2011). Advances and perspectives on the regulation and expression of piscine heat shock proteins. Rev. Fish Biol. Fish. 21, 153-185.

Fabra, M., Raldúa, D., Bozzo, M. G., Deen, P. M. T., Lubzens, E., and Cerdà, J. (2006). Yolk proteolysis and aquaporin-1o play essential roles to regulate fish oocyte hydration during meiosis resumption. Dev. Biol. 295, 250-262.
Fabra, M., Raldúa, D., Power, D. M., Deen, P. M. T., and Cerdà, J. (2005). Marine fish egg hydration is aquaporin-mediated. Science 307, 545.

Gaitskell, R. E., and Chester Jones, I. (1970). Effects of adrenalectomy and cortisol injection on the in vitro movement of water by the intestine of the freshwater European eel (Anguilla anguilla L.). Gen. Comp. Endocrinol. 15, 491-493.

Giffard-Mena, I., Boulo, V., Aujoulat, F., Fowden, H., Castille, R., and Charmantier, G. (2007). Aquaporin molecular characterization in the sea-bass (Dicentrarchus labrax): the effect of salinity on AQP1 and AQP3 expression. Comp. Biochem. Physiol. 148A, 430-444.

Heymann, J. B., and Engel, A. (1999). Aquaporins: phylogeny, structure, and physiology of water channels. News Physiol. Sci. 14, 187-193.

Hirano, T., and Utida, S. (1968). Effects of ACTH and cortisol on water movement in isolated intestine of the eel, Anguilla japonica. Gen. Comp. Endocrinol. 11, 373-380.

Hirata, T., Kaneko, T., Ono, T., Nakazato, T., Furukawa, N., Hasegawa, S., Wakabayashi, S., Shigekawa, M., Chang, M. H., Romero, M. F., and Hirose, S. (2003). Mechanism of acid adaptation of a fish living in a pH 3.5 lake. Am. J. Physiol. 284, 1199-1212.

Ishibashi, K., Hara, S., and Kondo, S. (2009). Aquaporin water channels in mammals. Clin. Exp. Nephrol. 13, $107-117$. 
Jung, J. S., Preston, G. M., Smith, B. L., Guggino, W. B., and Agre, P. (1994). Molecular structure of the water channel through aquaporin CHIP. The hourglass model. J. Biol. Chem. 269, 14648-14654.

Kelly, S. P., and Woo, N. Y. S. (1999). The response of sea bream during hyposmotic exposure. J. Fish Biol. 55, 732-750.

Lignot, J. H., Cutler, C. P., Hazon, N., and Cramb, G. (2002). Immunolocalisation of aquaporin 3 in the gill and the gastrointestinal tract of the European eel Anguilla anguilla (L.). J. Exp. Biol. 205, 2653-2663.

Liu, H., Hooper, S. B., Armugam, A., Dawson, N., Ferraros, T., Jeyaseelan, K., Theil, A., Koukoulas, I., and Wintour, E. M. (2003). Aquaporin gene expression and regulation in the ovine fetal lung. J. Physiol. 551, 503-514.

Mancera, J. M., Fernández-Llebrez, P., and Pérez-Figares, J. M. (1995). Effect of decreased environmental salinity on growth hormone cells in the euryhaline gilthead seabream (Sparus aurata L.). J. Fish Biol. 46, 494-500.

Martinez, A. S., Cutler, C. P., Wilson, G. D., Philips, C., Hazon, N., and Cramb, G. (2005a). Cloning and expression of three aquaporin homologues from the European eel (Anguilla anguilla): effects of seawater acclimation and cortisol treatment on renal expression. Biol. Cell 97, 615-627.

Martinez, A. S., Cutler, C. P., Wilson, G. D., Philips, C., Hazon, N., and Cramb, G. (2005b). Regulation of expression of two aquaporin homologues in the intestine of the European eel: effects of seawater acclimation and cortisol treatment. Am. J. Physiol. 57, 1733-1743.

Martinez, A. S., Wilson, G. D., Philips, C., Cutler, C. P., Hazon, N., and Cramb, G. (2005c). Effect of cortisol on aquaporin expression in the esophagus of the European eel,
Anguilla anguilla. Ann. N. Y. Acad. Sci. 1040, 395-398.

McCormick, S. D. (1995). "Hormonal control of gill $\mathrm{Na}^{+}-\mathrm{K}^{+}$-ATPase and chloride cell function," in Cellular and Molecular Approaches to Fish Ionic Regulation, eds C. M. Wood and T. J. Shuttleworth (New York: Academic Press), 285-315.

McDonald, M. D., and Grosell, M. (2006). Maintaining osmotic balance with an aglomerular kidney. Comp. Biochem. Physiol. 143A, 447458.

Mommsen, T. P., Vijayan, M. M., and Moon, T. W. (1999). Cortisol in teleosts: dynamics, mechanisms of action and metabolic regulation. Rev. Fish Biol. Fish 9, 211-268.

Motais, R., and Isaia, J. (1972). Temperature-dependence of permeability to water and to sodium of the gill epithelium of the eel Anguilla anguilla. J. Exp. Biol. 56, 587-600.

Nishimura, H., and Imai, M. (1982). Control of renal function in freshwater and marine teleosts. Fed. Proc. 41, 2355-2360.

Ogasawara, T., and Hirano, T. (1984). Changes in osmotic water permeability of the eel gills during seawater and freshwater adaptation. J. Comp. Physiol. B 154, 3-11.

Pelis, R., and McCormick, S. D. (2001). Effects of growth hormone and cortisol on $\mathrm{Na}^{+}-\mathrm{K}^{+}-2 \mathrm{Cl}^{-}$cotransporter localization and abundance in the gills of Atlantic salmon. Gen. Comp. Endocrinol. 124, 134-143.

Raldúa, D., Otero, D., Fabra, M., and Cerdà, J. (2008). Differential localization and regulation of two aquaporin-1 homologs in the intestinal epithelia of the marine teleost Sparus aurata. Am. J. Physiol. 294, 993-1003.

Sakamoto, T., and McCormick, S. D. (2006). Prolactin and growth hormone in fish osmoregulation. Gen. Comp. Endocrinol. 147, 24-30.

Sangio-Alvarellos, S., Polakof, S., Arjona, F. J., Kleszczynska, A., del Río, M. P. M., Míguez, J. M., Soengas, J. L., and Mancera, J. M. (2006). Osmoregulatory and metabolic changes in the gilthead sea bream Sparus auratus after arginine vasotocin (AVT) treatment. Gen. Comp. Endocrinol. 148, 348-358.

Shi, L. B., Skach, W. R., and Verkman, A. S. (1994). Functional independence of monomeric CHIP28 water channels revealed by expression of wildtype mutant heterodimers. J. Biol. Chem. 269, 10417-10422.

Stoenoiu, M. S., Ni, J., Verkaeren, C., Debaix, H., Jona, J. C., Lamiere, N., Verbavatz, J. M. and Devuyst, O. (2003). Corticosteroids induce expression of aquaporin1 and increase transcellular water transport in rat peritoneum. J. Am. Soc. Nephrol. 14 555-565.

Tanaka, M., Inase, N., Fushimi, K., Ishibashi, K., Ichioka, M., Sasaki, S., and Marumo, F. (1997). Induction of aquaporin 3 by corticosteroid in a human airway epithelial cell line. Am. J. Physiol. 273, L1090-L1095.

Tingaud-Sequeira, A., Chauvigné, F., Fabra, M., Lozano, J., Raldúa, D. and Cerdà, J. (2008). Structural and functional divergence of two fish aquaporin-1 water channels following teleost-specific gene duplication. BMC Evol. Biol. 8, 259-277. doi: 10.1186/1471-2148-8-259

Tipsmark, C. K., Sørensen, K. J., and Madsen, S. S. (2010). Aquaporin expression dynamics in osmoregulatory tissues of Atlantic salmon during smoltification and seawater acclimation. J. Exp. Biol. 213, 368-379.

Verkman, A. S., and Mitra, A. K. (2000). Structure and function of aquaporin water channels. Am. J. Physiol. 278, F13-F28.

Virkki, L. V., Cooper, G. J., and Boron, W. F. (2001). Cloning and functional expression of a MIP (AQP0) homolog from killifish (Fundulus heteroclitus) lens. Am. J. Physiol. 281, R1994-R2003.

Watanabe, S., Kaneko, T., and Aida, K. (2005). Aquaporin-3 is expressed in the basolateral membrane of gill chloride cells in Mozambique tilapia Oreochromis mossambicus adapted to freshwater and seawater. J. Exp. Biol. 208, 2673-2682.

Wintour, E. M., Earnest, L., Alcorn, D., Butkus, A., Shandley, L. and Jeyaseelan, K. (1998). Ovine AQP1: cDNA cloning, ontogeny and control of renal gene expression. Pediatr. Nephrol. 12, 545-553.

Woo, N. Y. S., and Kelly, S. P. (1995). Effects of salinity and nutritional status on growth and metabolism of Sparus sarba in a closed seawater system. Aquaculture 135, 229-238.

Zardoya, R. (2005). Phylogeny and evolution of the major intrinsic protein family. Biol. Cell 97, 397-414.

Conflict of Interest Statement: The authors declare that the research was conducted in the absence of any commercial or financial relationships that could be construed as a potential conflict of interest.

Received: 19 April 2011; accepted: 09 July 2011; published online: 21 July 2011. Citation: Deane EE, Luk JCY and Woo NYS (2011) Aquaporin 1a expression in gill, intestine, and kidney of the euryhaline silver sea bream. Front. Physio. 2:39. doi: 10.3389/fphys.2011.00039

This article was submitted to Frontiers in Aquatic Physiology, a specialty of Frontiers in Physiology.

Copyright $\odot 2011$ Deane, Luk and Woo. This is an open-access article subject to a non-exclusive license between the authors and Frontiers Media SA, which permits use, distribution and reproduction in other forums, provided the original authors and source are credited and other Frontiers conditions are complied with. 